
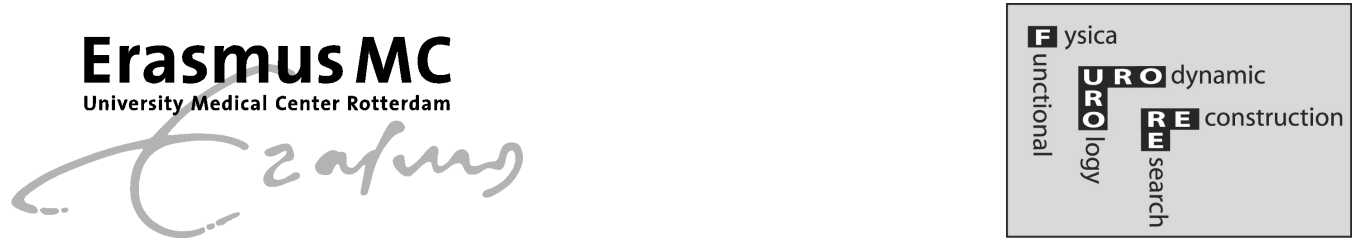

\title{
A biophysical model of the male urethra: comparing viscoelastic properties of PolyVinyl Alcohol urethras to male pig urethras.
}

\author{
Authors: \\ Tim Idzenga, Johan J.M. Pel and Ron van Mastrigt \\ ErasmusMC, Dept. of Urology, Sector Furore, Rotterdam, The Netherlands
}

Correspondence to:

Tim Idzenga

Erasmus MC, dept. of Urology

Sector Furore, room EE1630

PO Box 1738

3000 DR, Rotterdam, The Netherlands

Tel: **-31-10-4087379

Fax: ${ }^{* *}-31-10-4089451$

t.idzenga@erasmusmc.nl 


\begin{abstract}
Aims: We aim at developing a non-invasive method for grading and diagnosing urinary bladder outlet obstruction, based on noise recording with a perineal contact microphone during voiding. We found that the noise production during voiding depends amongst others on the viscoelastic properties of the urethral wall. To further test our method, we need a realistic biophysical model of the male urethra. Methods: We made various model urethras with different viscoelastic properties from a $10 \%$ aqueous solution of PolyVinyl Alcohol cryogel. We measured the viscoelastic properties of each model and compared them to those of the male pig urethra. The male pig urethra was used, as it is physiologically comparable to the human male urethra. The viscoelastic properties of both model and pig urethras were measured by applying strain to the urethral wall in a stepwise manner and recording the pressure response. We fitted the step-response of a mechanical model to this pressure response and derived the viscoelastic properties from the coefficients of this response. Results: A uniform model urethra that was freeze-thawed three times, with a Y-shaped flow channel was found to best represent the male pig urethra. Conclusion: We consider the three times freeze-thawed model urethra with a Y-shaped flow channel the best model of the human male urethra. And we therefore use this model urethra for studying the relation between noise recording during urine flow and the degree of bladder outlet obstruction.
\end{abstract}




\section{Introduction}

At present a pressure-flow study is the standard method used in urology to diagnose urinary bladder outlet obstruction. An enlarged prostate may cause such an obstruction. For a pressure-flow study a catheter is inserted via the urethra into the bladder. During voiding the urinary flow-rate and the pressure in the bladder are measured and the combination of these two indicates whether the bladder outlet is obstructed or not. This method is time-consuming, and it may also induce urethral trauma (Klingler et al. 1998, Porru et al. 1999). We are developing an alternative non-invasive method that is based on the turbulent flow-pattern in the male urethra during voiding (Idzenga et al. 2005). This flow pattern causes pressure fluctuations at the urethral wall that can be measured by placing a piezoelectric microphone against the perineum, between scrotum and anus (Bradley et al. 1977, Koiso et al. 1991).

In recent decades the relationship between audible noise and the degree of obstruction has been studied with the aim of diagnosing cardiovascular stenosis (Duncan et al. 1975, Kistler et al. 1981) and bladder outlet obstruction (Koiso et al. 1991, van Koeveringe \& van Mastrigt 1991). Latex tubing (Jones \& Fronek 1987, Tobin \& Chang 1976) has been used as a model of blood vessels, and silicone tubing to model the urethra (Teriö 1991). However, these types of tubing are considerably stiffer than arteries and the male urethra. A more flexible and extensible tube can be made from a Polyvinyl Alcohol (PVA) cryogel (Chu \& Rutt 1997). The number of freeze/thaw cycles, the rate of freezing/thawing and the concentration of the cryogel control the viscoelastic properties of this PVA cryogel. For studying the relationship between noise and degree of obstruction, such a PVA tube could be used as a realistic model of the male urethra.

To establish which model urethra best represents the male urethra we have measured viscoelastic properties of different model urethras and tuned these to those measured in pig urethras. To this end, we varied the number of freeze-thaw cycles, the profile of the flow channel in the model and the number of cross-sectional layers. The rate of freezing/thawing and the concentration of the PVA cryogel were the same in all models. We used the male pig urethra as a reference for the development of a model urethra because of the similarities between pig and human physiology (Douglas 1972). To measure the viscoelastic properties we used the method developed by Coolsaet et al. (Coolsaet et al. 1973, Coolsaet, van Duyl, van Mastrigt \& Schouten 1975, Coolsaet, van Duyl, van Mastrigt \& van der Zwart 1975), adapting it for use in cylindrical structures. This method involves the stepwise application of strain to the urethral wall by rapidly injecting known volumes of water into the urethra and recording the pressure response. 


\section{Materials \& Methods}

We made 12 different model urethras by pouring a $10 \%$ aqueous solution of PVA into a cylindrical mould. After 6 hours of rest at room temperature $\left(21^{\circ} \mathrm{C}\right)$, the mould was stored in a freezer at $-20^{\circ} \mathrm{C}$. After 14 hours in the freezer the mould was stored at room temperature for 10 hours. That completed one freeze-thaw cycle. To create a channel allowing flow through the models, we placed either a strip with a Y-profile (with legs $5 \mathrm{~mm}$ wide) or a circular rod (diameter $4 \mathrm{~mm}$ ) along the central axis of the mould (see Figure 1 .

The Y-profile reflects the lumen of a male pig urethra without flow of urine (see Figure 22 and the circular profile reflects the expected profile of a male pig urethra during urine flow. Four models were uniform solid cylinders (outer diameter $16 \mathrm{~mm}$ ) with a Y-profile; these were freeze-thawed one, two, three and four times respectively (indicated as $\mathrm{Y}_{1}, \mathrm{Y}_{2}, \mathrm{Y}_{3}$ and $\mathrm{Y}_{4}$ ). Four more models were also uniform solid cylinders (outer diameter $16 \mathrm{~mm}$ ) and freeze-thawed one, two, three and four times but these had a circular profile (indicated as $\mathrm{O}_{1}, \mathrm{O}_{2}, \mathrm{O}_{3}$ and $\mathrm{O}_{4}$ ). Another four models consisted of cylinders similar to those described above (but with an outer diameter of $12 \mathrm{~mm}$ ) inside a separate shell. Two of the cylinders had a Y-profile and two had a circular profile. For each profile one cylinder was freeze-thawed

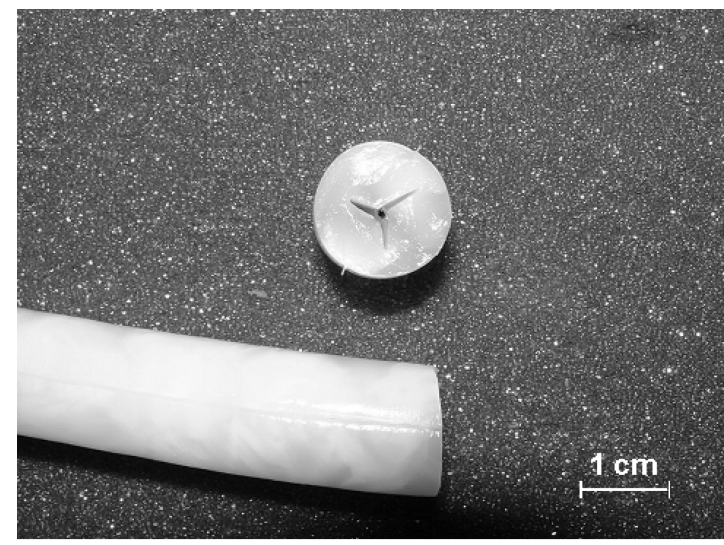
once the other was freeze-thawed twice. All four cylinders were placed in a PVA shell (inner diameter $12 \mathrm{~mm}$, outer diameter $16 \mathrm{~mm}$ ) that was freeze-thawed three times. These models are indicated as $\mathrm{Y}_{1 / 3}, \mathrm{Y}_{2 / 3}, \mathrm{O}_{1 / 3}$ and $\mathrm{O}_{2 / 3}$. Typical pressure-flow curves for model urethras with a Y-shaped profile and with a circular profile are shown in Figure 3. In the human urethra hysteresis causes the pressure flow curves to take the form of a loop. This is by variation in the degree of urethral relaxation during voiding. In the model urethras no hysteresis loop is observed, as there is no such relaxation mechanism.

We made bladder/urethra preparations from 8 male pigs sacrificed at the department of Experimental Cardiology (Erasmus MC, Rotterdam, the Netherlands). The part of the urethra closest to the bladder (proximal part), distal to the prostate was selected for the measurements. The prostate of the pig is located just under the bladder. The average $( \pm 1 \mathrm{sd})$ length of the sections was $65 \pm 16 \mathrm{~mm}$. An example of a cross-section is shown in Figure 2, We used Elastic von Gieson staining (Bancroft \& Stevens 1996) to mark elastin, collagen and muscle tissue in this cross-section. 


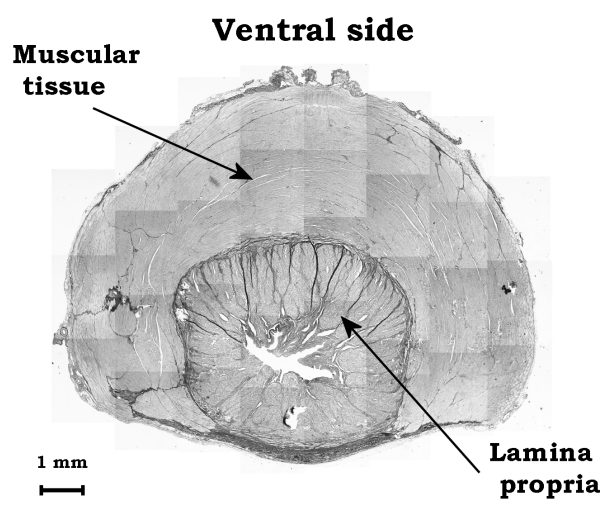

Figure 2: Cross-section of the proximal part of the male pig urethra. We stained elastin, collagen (both dark grey) and muscle tissue (light grey) using Elastic von Gieson staining. The lamina propria is surrounded by muscular tissue with a horseshoe-like shape.

$0.2 \mathrm{ml}$ to a maximum of $5 \mathrm{ml}$, or to the volume at which the ra transducer $\left(\sim 250 \mathrm{~cm} \mathrm{H}_{2} \mathrm{O}\right)$ was exceeded. After each measurement the injected water was extracted. The transducer signal was sampled at a frequency of $1000 \mathrm{~Hz}$ using an AD-converter (PCL-818, Advantech ${ }^{\circledR}$ ) and stored in a PC for further analysis using custom-written programs in Matlab ${ }^{\circledR}$.

For an approximate calculation of the viscoelastic properties of the model and pig urethras from the recorded pressure response we made a mechanical model of the urethral wall consisting of springs, dashpots and a mass (see Appendix). The simplified analytical solution for the stepresponse (Eqn. A - 6) was fitted to 1000 samples (equivalent of $1 \mathrm{sec}$.) of the pressure signal. These 1000 samples were selected from the maximum in the pressure signal onwards (see Figure 4 and 5). The fit-procedure resulted in 6 coefficients: $a_{0,1,2}, \quad b_{1,2}$ and $c_{2}$. The coefficients $a_{0,1,2}$ were converted from units of pressure into units of stress $\left(\sigma_{0,1,2}\right)$ using Laplaces Law (Coolsaet, van Duyl, van Mastrigt \& van der Zwart 1975), see Appendix. The coefficients
Each model was placed in a water-filled container (approx. $1 \mathrm{~cm}$ below the watersurface) at room temperature and each pig urethra was placed in a container (approx. $1 \mathrm{~cm}$ below the solution-surface) filled with a cold $\left(10^{\circ} \mathrm{C}\right)$ modified Krebs solution $(118 \mathrm{mM}$ $\mathrm{NaCl} ; 4.7 \mathrm{mM} \mathrm{KCl} ; 25 \mathrm{mM} \mathrm{NaHCO} 3 ; 1.2 \mathrm{mM}$ $\mathrm{KH}_{2} \mathrm{PO} 4 ; 1.8 \mathrm{mM} \mathrm{CaCl}_{2} ; 1.2 \mathrm{mM} \mathrm{MgSO}$ and $11 \mathrm{mM}$ glucose aerated with $95 \% \mathrm{O}_{2}$ and $5 \%$ $\mathrm{CO}_{2}$ (pH of 7.4)). One side of the model or pig urethra was connected to a $5 \mathrm{ml}$ syringe to manually inject a known volume of water in a very short time. The pressure response was recorded using a disposable pressure transducer connected to the other side of the urethra. The pressure transducer was zeroed to the atmosphere at the level of the pig or model urethra. Increasing volumes were involume at which the range of the pressure
voluting at $0.2 \mathrm{ml}$ with increments of

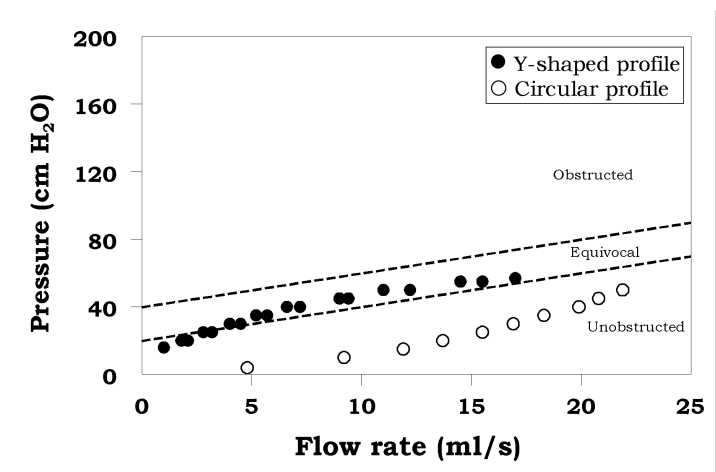

Figure 3: Typical pressure-flow curve for unobstructed model urethras with a Y-shaped profile and with a circular profile. The flow rate is measured at discreet values of driving pressure. The dotted lines discriminate unobstructed, equivocal and obstructed curves according to the provisional ICS method for diagnosing infravesical obstruction.

$b_{1}$ and $b_{2}$ were converted into the time-constants $\tau_{1}$ and $\tau_{2}$ (the reciprocals of $b_{1}$ and $\mathrm{b}_{2}$ ). The oscillation frequency $f$ of the mass in the mechanical model was derived from $\mathrm{c}_{2}$.

Differences in the three time-related parameters (i.e. $\tau_{1}, \tau_{2}$ and $f$ ) between each model urethra and the pig urethras were tested using a Mann-Whitney U-test. The stress-parameters (i.e. $\sigma_{0}, \sigma_{1}$ and $\sigma_{2}$ ) in pig urethras were found to depend on the applied strain. The stress-strain functions of each pig urethra were fitted with an exponential function and the average exponential function and its approximate $95 \%$ Confidence Interval (CI) were calculated. As a measure of agreement, we used the overlap between the stress-parameters measured in the models and the $95 \%$ CI calculated for the pig urethras. 


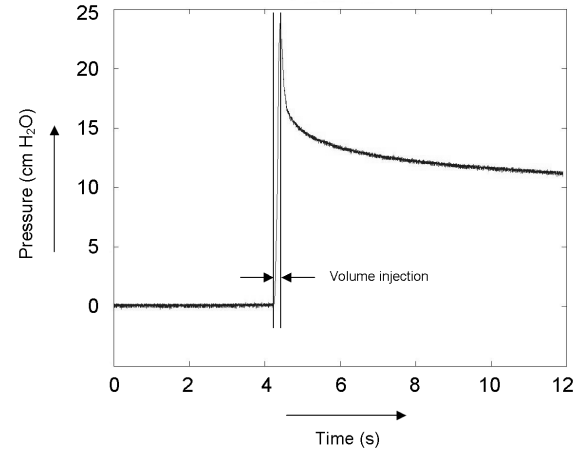

Figure 4: Example of the pressure response of a pig urethra to stepwise straining by injecting a volume of water. The step response of the mechanical model was fitted to a 1000 samples (equivalent to 1 second of the pressure signal) from the maximum pressure onwards.
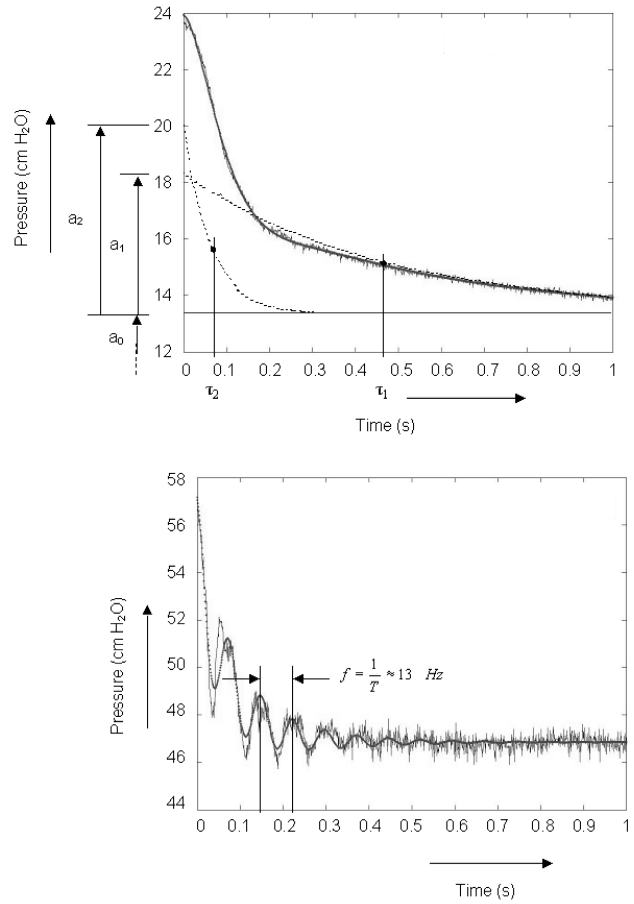

Figure 5: Examples of the selected part of the measured pressure response and the fitted function in a pig urethra (top panel) and model urethra $\mathrm{Y}_{2}$ (lower panel). The solid line represents the analytical solution of the mechanical model (with a low frequent oscillation of appr. $2 \mathrm{~Hz}$ ) fitted to the recorded data. In the top panel the two dotted lines represent the two exponential terms without taking the multiplicative oscillation term into account. The amplitudes and time-constants of the exponentials are indicated by $\mathrm{a}_{0,1,2}$ and $\tau_{t 1,2}$. The bottom panel shows a high frequent oscillation in one of the model urethras. 


\section{Results}

An example of the pressure response to injection of water in a pig urethra is shown in Figure 4. The injection time was kept short $(\sim 0.1 \mathrm{~s})$ to induce a step-response. Figure 5, top panel, shows the selected part of the pressure response measured in a pig urethra. Upon injection of the volume the pressure increased rapidly and then decreased bi-exponentially $\left(\tau_{1}\right.$ and $\tau_{2}$ ) to an equilibrium pressure. In the bottom panel the pressure response measured in a model urethra is shown. Again, pressure decreased bi-exponentially, but in most of the models the pressure signal showed a more distinct oscillation $(f)$ than in pig urethras. The wall of the model with a Y-shaped channel that was freeze-thawed one time was not strong enough to withstand the induced stress.

Table I summarizes the mean $( \pm \mathrm{SD})$ values of $f, \tau_{1}$ and $\tau_{2}$ measured in pig and model urethras, including the results of the MannWhitney U-test. Figure 6 shows the oscillation frequency measured in the male pig urethras and in some of the models with different crosssectional layers, number of freeze-thaw cycles and channel shapes. The models for which $f$ was not significantly different from that measured in the pig urethras were $\mathrm{Y}_{4}$ and $\mathrm{O}_{3}$. In some of the models a discontinuity in the frequency of oscillation was observed at a certain level of strain: $\mathrm{Y}_{3}$ (at $\epsilon \sim 0.37$ ), $\mathrm{Y}_{4}$ (at $\epsilon \sim 0.2$ ), $\mathrm{O}_{2}$ (at $\epsilon \sim 0.15$ ) and $\mathrm{O}_{2 / 3}$ (at $\epsilon \sim 0.18$ ). The models with an oscillation frequency that was not significantly different from that in male pig urethras, but only at the higher levels of strain were: $\mathrm{Y}_{3}, \mathrm{O}_{2}$ and $\mathrm{O}_{2 / 3}$.

\begin{tabular}{|c|c|c|c|c|}
\hline & $\mathrm{N}$ & $\tau_{1}(\mathbf{s})$ & $\tau_{2}(\mathbf{s})$ & $f(\mathrm{~Hz})$ \\
\hline $0^{\pi}$ & 88 & $0.47 \pm 0.30$ & $0.046 \pm 0.026$ & $2.9 \pm 1.8$ \\
\hline $\mathrm{Y}_{2}$ & 20 & $0.09 \pm 0.06(\mathbf{p}<\mathbf{. 0 0 1})$ & $0.141 \pm 0.055(\mathbf{p}<\mathbf{. 0 0 1})$ & $12.4 \pm 3.5(\mathbf{p}<\mathbf{. 0 0 1})$ \\
\hline $\mathrm{Y}_{3}$ & 19 & $0.40 \pm 0.25(\mathbf{p}=\mathbf{. 7 3 5})$ & $0.054 \pm 0.015(\mathbf{p}<\mathbf{. 0 5 0})$ & $6.6 \pm 5.9(\mathbf{p}<. \mathbf{0 1 0})$ \\
\hline $\mathrm{Y}_{4}$ & 16 & $0.41 \pm 0.14(\mathbf{p}=.690)$ & $0.062 \pm 0.016(\mathbf{p}<\mathbf{. 0 0 1})$ & $4.9 \pm 5.1(\mathbf{p}=. \mathbf{2 1 5})$ \\
\hline $\mathrm{Y}_{1 / 3}$ & 24 & $0.07 \pm 0.03(\mathbf{p}<\mathbf{. 0 0 1})$ & $0.161 \pm 0.040(\mathbf{p}<\mathbf{. 0 0 1})$ & $7.2 \pm 1.8(\mathbf{p}<. \mathbf{0 0 1})$ \\
\hline $\mathrm{Y}_{2 / 3}$ & 24 & $0.17 \pm 0.15(\mathbf{p}<\mathbf{. 0 0 1})$ & $0.105 \pm 0.044(\mathbf{p}<\mathbf{. 0 0 1})$ & $12.1 \pm 5.8(\mathbf{p}<. \mathbf{0 0 1})$ \\
\hline $\mathrm{O}_{1}$ & 24 & $0.12 \pm 0.05(\mathbf{p}<\mathbf{. 0 0 1})$ & $0.216 \pm 0.036(\mathbf{p}<\mathbf{. 0 0 1})$ & $10.7 \pm 0.9(\mathbf{p}<\mathbf{. 0 0 1})$ \\
\hline $\mathrm{O}_{2}$ & 18 & $0.38 \pm 0.32(\mathbf{p}=. \mathbf{1 4 1})$ & $0.093 \pm 0.045(\mathbf{p}<\mathbf{. 0 0 1})$ & $10.2 \pm 8.3(\mathbf{p}<. \mathbf{0 1 0})$ \\
\hline $\mathrm{O}_{3}$ & 7 & $0.46 \pm 0.01(\mathbf{p}=. \mathbf{4 2 8})$ & $0.075 \pm 0.007(\mathbf{p}<\mathbf{. 0 0 1})$ & $2.4 \pm 0.05(\mathbf{p}=. \mathbf{7 4 3})$ \\
\hline $\mathrm{O}_{4}$ & 5 & $0.20 \pm 0.01(\mathbf{p}<\mathbf{. 0 0 1})$ & $0.122 \pm 0.013(\mathbf{p}<\mathbf{. 0 0 1})$ & $12.7 \pm 0.8(\mathbf{p}<\mathbf{. 0 0 1})$ \\
\hline $\mathrm{O}_{1 / 3}$ & 24 & $0.09 \pm 0.04(\mathbf{p}<\mathbf{. 0 0 1})$ & $0.123 \pm 0.013(\mathbf{p}<\mathbf{. 0 0 1})$ & $12.1 \pm 1.7(\mathbf{p}<\mathbf{. 0 0 1})$ \\
\hline $\mathrm{O}_{2 / 3}$ & 17 & $0.30 \pm 0.19(\mathbf{p}<\mathbf{. 0 5 0})$ & $0.099 \pm 0.052(\mathbf{p}<\mathbf{. 0 0 1})$ & $12.5 \pm 7.5(\mathbf{p}<. \mathbf{0 0 1})$ \\
\hline
\end{tabular}

Table 1: Time-parameters $f, \tau_{1}$ and $\tau_{2}$ (mean $\pm \mathrm{SD}$ ) and the number of measurements that were done in the male pig urethra (indicated by $o^{7}$ ) and in the different model urethras. The results of the Mann-Whitney U-tests are noted by the p-values between brackets.

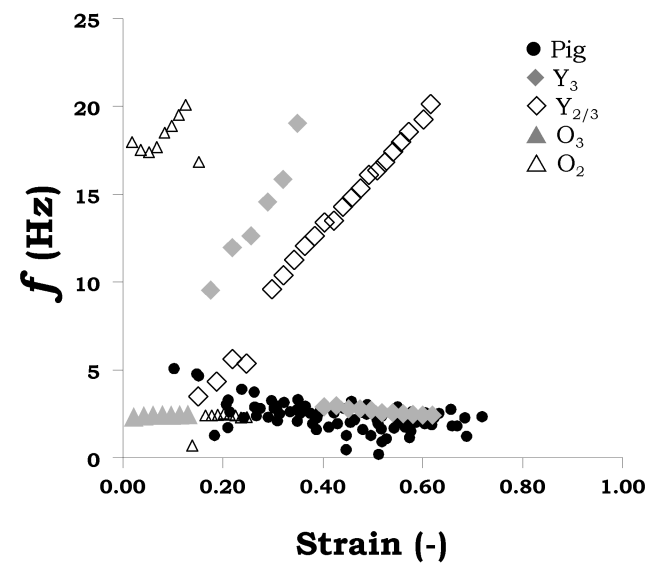

Figure 6: Oscillation frequency $f$ as a function of the applied strain measured in male pig urethras and in model urethras $\mathrm{Y}_{3}, \mathrm{Y}_{2} / 3, \mathrm{O}_{2}$ and $\mathrm{O}_{3}$. The oscillation frequency in model $\mathrm{O} 3$ was found not to be significantly different from the frequencies measured in the male pig urethra. $\mathrm{Y}_{3}$ is an example of a model in which the oscillation frequency equalled that of the pig urethras, but only at higher strain levels. $\mathrm{Y}_{2 / 3}$ shows oscillation frequencies higher than those meaoscillation frequencies higher tha
sured in the male pig urethras. 


\begin{tabular}{l|c|c|c|c|c|c} 
& $\tau_{1}$ & $\tau_{2}$ & $f$ & $\sigma_{0}$ & $\sigma_{1}$ & $\sigma_{2}$ \\
\hline \hline $\mathrm{Y}_{2}$ & - & - & - & - & - & - \\
$\mathrm{Y}_{3}$ & $\mathbf{+}$ & $\mathbf{+}$ & \pm & - & $\mathbf{+}$ & $\mathbf{+}$ \\
$\mathrm{Y}_{4}$ & $\mathbf{+}$ & $\mathbf{+}$ & $\mathbf{+}$ & - & - & $\mathbf{+}$ \\
$\mathrm{Y}_{1 / 3}$ & - & - & - & \pm & - & \pm \\
$\mathrm{Y}_{2 / 3}$ & - & - & - & - & \pm & - \\
$\mathrm{O}_{1}$ & - & - & - & - & $\mathbf{+}$ & $\mathbf{+}$ \\
$\mathrm{O}_{2}$ & $\mathbf{+}$ & \pm & \pm & - & - & - \\
$\mathrm{O}_{3}$ & $\mathbf{+}$ & - & $\mathbf{+}$ & - & - & - \\
$\mathrm{O}_{4}$ & - & - & - & - & - & - \\
$\mathrm{O}_{1 / 3}$ & - & - & - & - & - & - \\
$\mathrm{O}_{2 / 3}$ & \pm & \pm & \pm & - & - & - \\
\hline
\end{tabular}

Table 2: Comparison of viscoelastic parameters between the different model urethras and the male pig urethra. The agreement between the male pig urethra and the different model urethras is depicted as good $(+)$, adequate $( \pm$ ) or bad $(-)$.

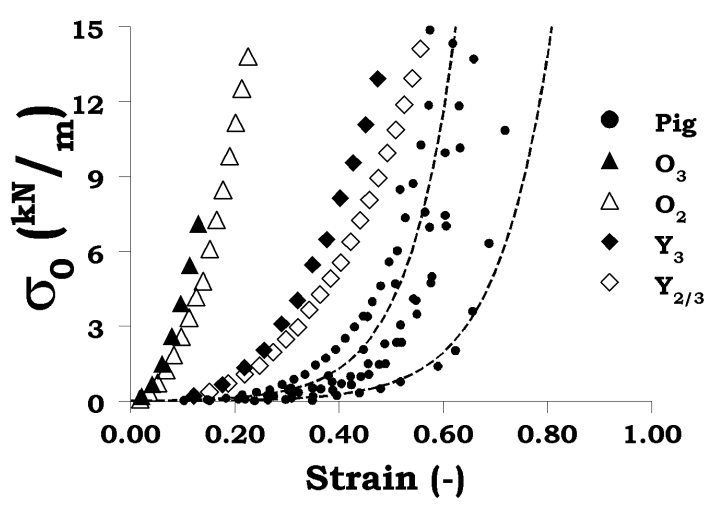

Figure 7: Stress-strain graphs of $\sigma_{0}$-values measured in the male pig urethra and in model urethras $\mathrm{Y}_{3}, \mathrm{Y}_{2 / 3}, \mathrm{O}_{2}$ and $\mathrm{O}_{3}$. The solid lines represent the average of the exponential functions fitted to the stressstrain curves of the male pig urethra. The dotted lines represent the $95 \% \mathrm{CI}$ of the fitted function.
The top panel of Figure 9 shows the time-constant $\tau_{1}$ measured in the pig urethras and some of the model urethras. The models for which $\tau_{1}$ was not significantly different from $\tau_{1}$ in the male pig urethra were: $\mathrm{Y}_{3}, \mathrm{Y}_{4}, \mathrm{O}_{2}$ and $\mathrm{O}_{3}$. The models that showed a discontinuity in $f$ also showed a discontinuity in $\tau_{1}$ at the same level of strain.

All model urethras had a time-constant $\tau_{2}$ that was significantly different from that in the male pig urethra. However, the model with $\tau_{2}$ closest to the one measured in male pig urethras was $\mathrm{Y}_{3}(\mathrm{p}<.05)$, see Table I and the bottom panel of Figure 9. Also in the time-constant $\tau_{2}$ the same four models showed a discontinuity at the same level of strain as for $f$ and $\tau_{1}$.

Figure 7 shows the stress-parameter $\sigma_{0}$ in the male pig urethra as a function of the applied strain and data of some model urethras with different cross-sectional layers, number of freeze-thaw cycles and channel shapes. The solid line represents the average of the exponential functions fitted to the data in each pig urethra and the dotted lines represent the $95 \%$ CI of this average. In the model urethras stress increased more rapidly as a function of strain with increasing number of freeze-thaw cycles. The increase in stress as a function of strain appeared to be higher in models with a circular opening than in model urethras with a Y-shaped opening. The stress-strain plots for the stress-parameters $\sigma_{1}$ and $\sigma_{2}$ were similar, except that the range of these stress-values was smaller than that of $\sigma_{0}$, see Figure 8 .

An overall comparison of the viscoelastic properties of the different model urethras and the male pig urethra, based on level of agreement, is presented in Table 2. From Table II it can be concluded that models $\mathrm{Y}_{3}$ and $\mathrm{Y}_{4}$ best represent the male pig urethra. 

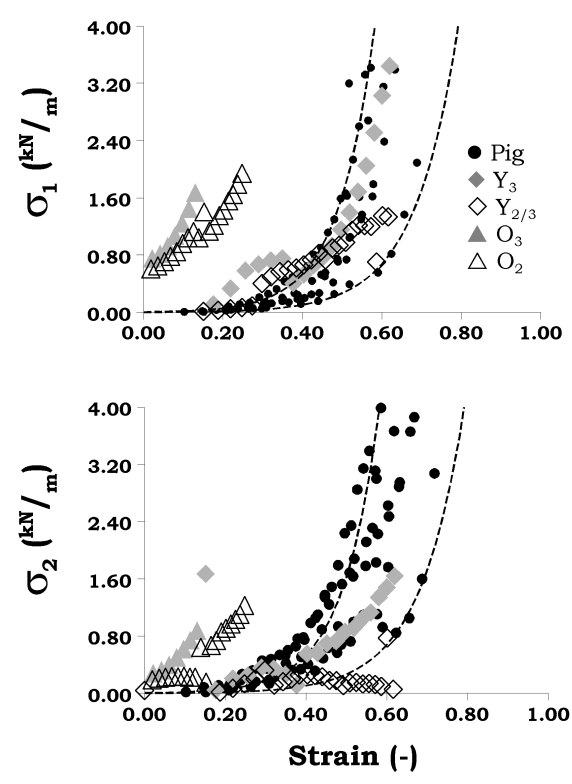

Figure 8: Stress-strain graphs of $\sigma_{1}$ - and $\sigma_{2}$ values measured in the male pig urethra and in model urethras $\mathrm{Y}_{3}, \mathrm{Y}_{2 / 3}, \mathrm{O}_{2}$ and $\mathrm{O}_{3}$. The solid lines represent the average of the exponential functions fitted to the stress-strain curves of the male pig urethra. The dotted lines represent the 95\% CI of the fitted function.
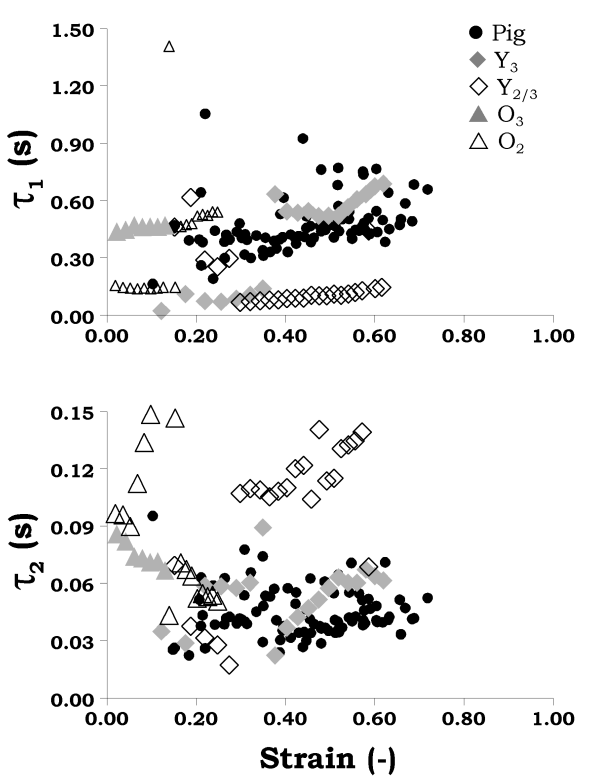

Figure 9: Time-constants in model urethras $\left(\mathrm{Y}_{3}\right.$, $\mathrm{Y}_{2 / 3}, \mathrm{O}_{2}$ and $\mathrm{O}_{3}$ ) and pig urethras. In the top panel $\tau_{1}$ and in the bottom panel $\tau_{2}$ is plotted against the applied strain. The scale of the Y-axis in the bottom panel is a factor 10 smaller than that of the top panel. Some of the models have a discontinuity in $\tau_{1}$ and $\tau_{2}$ at a certain level of strain, i.e. $\mathrm{Y}_{3}$ and $\mathrm{O}_{2}$.

\section{Discussion}

In order to study the relation between noise recorded at the perineum and urinary bladder outlet obstruction we have developed a biophysical model of the male urethra. To develop such a model we measured the viscoelastic properties of different model urethras made from PVA cryogel. The intended location of noise recording (i.e. perineum) is distal to the obstruction. Therefore, only the viscoelastic properties of the normal male urethra distal to the obstruction are relevant and we compared the properties of the model urethras to those measured in normal pig urethras. We varied three properties of the model urethras: the number of freeze-thaw cycles, the shape of the flow channel and the number of cross-sectional layers. It is known that during freezing and thawing the PVA solution forms cross-links through hydrogen bonding with hydroxyl groups on the PVA molecules (Chu \& Rutt 1997). When the number of freeze-thaw cycles is increased more cross-links are formed and thus the strength and stiffness increases. Also with increasing number of freeze-thaw cycles the PVA gets more and more compact and the outer diameter of the model urethra decreases slightly (appr. $1 \mathrm{~mm}$ ). This is not expected to significantly change the values of the parameters. The increase in stiffness with increasing number of freeze-thaw cycles was best demonstrated by the stress parameter $\sigma_{0}$ of the model urethras. With a larger number of freeze-thaw cycles the stress-parameter increased more rapidly with strain (which implies an increase in stiffness). The increase in stiffness with the number of freeze-thaw cycles was also found by Chu (Chu \& Rutt 1997). In Figure 7 only data of a few models has been plotted but this effect was found in all models. In models with a circular shaped opening the increase in stress, as a function of strain, appeared to be slightly higher than in models with a Y-shaped opening. In calculating the applied strain in models it was assumed that the 
injected volume in the model had a circular cross section. This is not exactly the case when the opening is Y-shaped initially. This might lead to a shift of the horizontal axis and cause the difference in stress-increase that was found. The models that consisted of two layers, one softer layer surrounded by a stiffer shell were thought to mimic the stress-strain plot of the pig urethras, i.e. a small increase in stress at low strain due to the soft inner layer and a steep increase in stress at high strain due to the stiffer shell. This however did not turn out to be the case. Application of strain to the soft inner layer by injecting a volume of water probably simultaneously applied strain to the outer shell, resulting in an interaction.

Viscoelastic properties of male pig urethras and model urethras were compared by fitting a mathematical model. When interpreting the estimated parameters it should be kept in mind that these are only one set of possible parameters in the modeling of the viscoelastic properties. The sensitivity of these parameters needs to be assessed in future studies. In the male pig urethras we found that the two time-constants $\tau_{1}$ and $\tau_{2}$ differed a factor of $\sim 10$ from each other, which supports the assumption that two independent relaxation processes (indicated by the two exponential terms, see Appendix) are activated after stepwise straining of the pig urethral wall. Four model urethras $\left(\mathrm{Y}_{3}\right.$, $\mathrm{Y}_{4}, \mathrm{O}_{2}$ and $\mathrm{O}_{3}$ ) had a time-constant $\tau_{1}$ that was not significantly different from that in male pig urethras. The agreement of $\tau_{1}$ between these four models and the male pig can therefore be considered as good. In all models the second time-constant $\tau_{2}$ measured in model urethras was statistically different from that of the male pig urethra. However, two model urethras with $\tau_{2}$ close to the range of values in male pig urethras were $\mathrm{Y}_{3}$ and $\mathrm{Y}_{4}$. Since the absolute difference between the $\tau_{2}$-means of pigs and models was quite small the agreement was considered good. There were two models with $\tau_{2}$ close to the range of values measured in pigs, but only at high strain: $\mathrm{O}_{2}$ and $\mathrm{O}_{2 / 3}$. The agreement of these models was considered adequate.

The oscillation frequency $f$ in most of the model urethras was higher than that in male pig urethras. In some of the models there was a discontinuity in the oscillation frequency with increasing strain: above a certain level of strain the frequency suddenly dropped to a lower level (e.g. see model $\mathrm{Y}_{3}$ in Figure 6). Such a discontinuity was also observed in the time-constants $\tau_{1}$ and $\tau_{2}$. At the lower level of strain the $\mathrm{t}$ - and $\sigma$-values of the second and third term of the step response (Eqn. A-6) are of approximately the same order (see Figure 9 and 8. At these levels of strain a high frequency oscillation (i.e. relative to the oscillation found in pig urethras) was found in the pressure response (see example in Figure 5). At the higher level of strain the $\tau$ - and $\sigma$-values of the second term of equation A-6 (see Appendix) are larger than those in the third term and therefore the oscillation in the third term was outdone by the second term. In models with a high oscillation frequency at each applied strain level the $\mathrm{t}$ - and $\sigma$-values of the two terms are approximately of the same order over the whole range of applied strain. Two models $\left(\mathrm{Y}_{4}\right.$ and $\left.\mathrm{O}_{3}\right)$ however had an oscillation frequency that was not significantly different from the male pig urethras over the full range of applied strains and we therefore considered the agreement between the frequency measured in these models and those in pigs to be good. Three model urethras $\left(\mathrm{Y}_{3}, \mathrm{O}_{2}\right.$ and $\left.\mathrm{O}_{2 / 3}\right)$ had an oscillation frequency not significantly different from the male pig urethras, in the higher region of applied strain only. Therefore, in these three models the agreement with pig urethras on the basis of the oscillation frequency is considered adequate.

The intended application of the model urethra we have developed is studying the relation between pressure fluctuations at the urethral wall, caused by a turbulent flow pattern, and the degree of bladder outlet obstruction. These pressure fluctuations are time-related and therefore the viscoelastic parameters $f, \tau_{1}$ and $\tau_{2}$ are the most important parameters in comparing the different models to the male pig urethra. Based on these parameters there are four models that best represent the male urethra, i.e. $\mathrm{O}_{2}$, $\mathrm{O}_{3}, \mathrm{Y}_{3}$ and $\mathrm{Y}_{4}$. When the associated amplitudes $\sigma_{1}$ and $\sigma_{2}$ are also taken into account only two models remain, i.e. $\mathrm{Y}_{3}$ and $\mathrm{Y}_{4}$. Adding up good and adequate agreement 
considerations model $\mathrm{Y}_{3}$ shows the best agreement with the male pig urethra.

\section{Conclusion}

We aim at developing a non-invasive method for grading and diagnosing urinary bladder outlet obstruction, based on noise recording with a perineal contact microphone during voiding. The recorded noise depends (among others) on the viscoelastic properties of the urethra. We therefore developed a biophysical model to study the relation between recorded noise and degree of bladder outlet obstruction. We measured the viscoelastic properties of different model urethras and compared them to the viscoelastic properties measured in male pig urethras. Based on this comparison a model urethra with a Y-shaped flow channel that was uniformly freeze-thawed three times showed best agreement with the male pig urethra. We therefore consider this model urethra the best model of the human male urethra for studying the relation between noise recording during urine flow and the degree of bladder outlet obstruction.

\section{Acknowledgement}

This research was supported by the Technology Foundation STW, applied division of the Netherlands Organization for Scientific Research (NWO) and the technology programme of the Ministry of Economic Affairs. 


\section{References}

Bancroft, J. \& Stevens, A. (1996), Theory and Practice of Histological Techniques, 4th edn, Churchill Livingstone.

Bradley, W., Brockway, B. \& Timm, G. (1977), 'Auscultation of urinary flow.', Journal of Urology 118, 73-75.

Chu, K. \& Rutt, B. (1997), 'Polyvinyl alcohol cryogel: an ideal phantom material for mr studies of flow and elasticity', MRM 37, 314-319.

Coolsaet, B., van Duyl, W., van Mastrigt, R. \& Schouten, J. (1975), 'Visoelastic properties of bladder wall strips', Investigative Urology 12(5), 351-356.

Coolsaet, B., van Duyl, W., van Mastrigt, R. \& van der Zwart, A. (1973), 'Step-wise cystometry of urinary bladder. new dynamic procedure to investigate viscoelastic behavior', Urology 2(3), 255-257.

Coolsaet, B., van Duyl, W., van Mastrigt, R. \& van der Zwart, A. (1975), 'Visco-elastic properties of the bladder wall', Urologia Internationalis 30(1), 16-26.

Douglas, W. (1972), 'Of pigs and men and research.', Space Life Sciences 3, 226-234.

Duncan, G., James, G., Forbes Dewey Jr, C., Myers, G. \& Lees, R. (1975), 'Evaluation of carotid stenosis by phonoangiography.', New England Journal of Medicine 27(Nov), 1124-1128.

Idzenga, T., Pel, J., Baldewsing, R. A. \& Mastrigt, R. (2005), 'Perineal noise recording as a non-invasive diagnostic method of urinary bladder outlet obstruction: a study in polyvinyl alcohol and silicone model urethras', Neurourol Urodyn 24(4), 381-8. Journal Article.

Jones, S. \& Fronek, A. (1987), 'Analysis of break frequency downstream of a constriction in a cylindrical tube.', Journal of Biomechanics 20(3), 319-327.

Kistler, J., Lees, R., Miller, A., Crowell, R. \& Roberson, G. (1981), 'Correlation of spectral phonoangiography and carotid angiography with gross pathology in carotid stenosis.', New England Journal of Medicine 305(8), 417-419.

Klingler, H., Madersbacher, S., Djavan, B., Schatzl, G., Marberger, M. \& Schmidbauer, C. (1998), 'Morbidity of the evaluation of the lower urinary tract with transurethral multichannel pressure-flow studies.', Journal of Urology 159(1), 191-194.

Koiso, K., Nemoto, R. \& Ohtani, M. (1991), 'Urographic studies of benign prostatic hypertrophy', Journal of Urology 145, 1071-1077.

Porru, D., Madeddu, G., Campus, G., Montisci, I., Scarpa, R. \& Usai, E. (1999), 'Evaluation of morbibidy of multi-channel pressure-flow studies.', Neurourology and Urodynamics 18(6), 647-652.

Teriö, H. (1991), 'Acoustic method for assessment of urethral obstruction: a model study', Medical \& Biological Engineering \& Computing 29, 450-456.

Tobin, R. \& Chang, I. (1976), 'Wall pressure spectra scaling downstream of stenoses in steady tube flow.', Journal of Biomechanics 9, 633-640.

van Koeveringe, A. \& van Mastrigt, R. (1991), 'A relation between the sound produced by urethral turbulence in patients and objectivel assessed subvesical obstruction. (abstract)'. 
van Mastrigt, R., Coolsaet, B. \& van Duyl, W. (1978), 'Passive properties of the urinary bladder in the collection phase.', Medical \& Biological Computing \& Engineering 16, 471-482. 


\section{Appendix}

The flow of urine through the urethra during voiding causes pressure-fluctuations on the urethral wall, which induce displacement. The urethral wall behaves like a flexible tube and has viscoelastic properties that affect the displacement. Viscoelasticity is a combination of both elastic and viscous properties of a material. Elasticity describes the relation between stress and strain and viscosity describes the resistance of a material to a change in form, i.e. the relation between stress and the change in strain. As a linear approximation of the viscoelastic properties of a urethra we have developed a mechanical model of the urethral wall consisting of springs, dashpots and a mass (see Figure 10.

A change in length $\ell$ of the model equally stretches each series of elements thereby generating a force in each series. These forces $(F)$ can be calculated from the general equations for each element:

$$
\begin{array}{ll}
F=E \cdot \ell & \text { (Spring) } \\
F=\eta \cdot \frac{\partial \ell}{\partial t} & \text { (Dashpot) } \\
F=m \cdot \frac{\partial^{2} \ell}{\partial t^{2}} & \text { (Mass) }
\end{array}
$$

With $E$ the elastic modulus of the spring, $\ell$ the length of each element, $\eta$ the viscosity of the dashpot and $m$ the mass of the mass element.

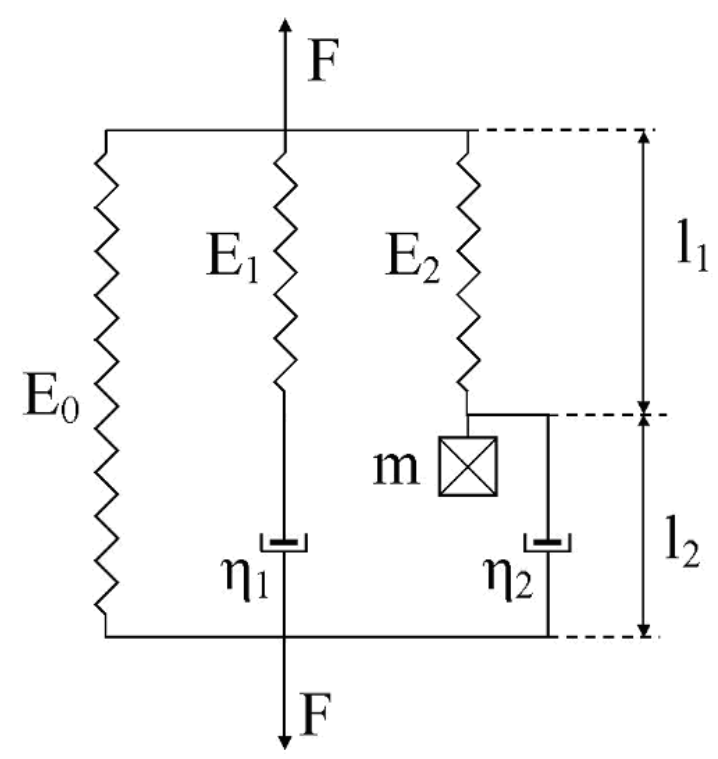

Figure 10: Mechanical model of the urethral wall consisting of springs $\left(E_{0,1,2}\right)$, dashpots $\left(\eta_{1,2}\right)$ and a mass $(m)$. The step-response function of this mechanical model was fitted to (a part of) the pressure-response of the model and pig urethras to stepwise application of strain.

When the model is strained stepwise:

$$
\ell=H(t) \cdot \Delta \ell \text {, with } H(t)=\left\{\begin{array}{ll}
0 & t<0 \\
1 & t \geq 0
\end{array}\right. \text { (Heaviside function) }
$$

with $\Delta \ell$ the change in length. 
From the Laplace-transformation of the sets of equations the Laplace-transform of the overall solution can be calculated. Inverse Laplace transformation of the overall solution results in an equation for the overall force that is generated by the mechanical model in response to stepwise straining:

$$
F(t)=\Delta \ell \cdot\left(E_{0}+E_{1} \cdot e^{-\frac{E_{1}}{\eta_{1}} \cdot t}+E_{2} \cdot e^{-\frac{\eta_{2}}{2 m} \cdot t} \cdot[g(t)]\right)
$$

With,

$$
g(t)=\cos \left(\sqrt{\frac{4 m E_{1}-\eta_{2}^{2}}{4 m^{2}}} \cdot t\right)+\frac{\eta_{2}}{\sqrt{4 m E_{1}-\eta_{2}^{2}}} \cdot \sin \left(\sqrt{\frac{4 m E_{1}-\eta_{2}^{2}}{4 m^{2}}}\right)
$$

This equation can be simplified for each length displacement of to:

$$
F(t)=a_{0}+a_{1} \cdot e^{-b_{1} \cdot t}+a_{2} \cdot e^{-b_{2} \cdot t} \cdot\left[\cos \left(c_{2} t\right)+\frac{b_{2}}{c_{2}} \cdot \sin \left(c_{2} t\right)\right]
$$

With:

- $a_{0}=$ equilibrium amplitude;

- $a_{1,2}=$ amplitudes of the exponential terms;

- $b_{1,2}=$ relaxation-constants of the exponential terms;

- $c_{2}=$ oscillation frequency.

In an idealized thin-walled urethra the tension $T$ in the urethral wall can be calculated from the recorded pressure response of the urethra using Laplaces Law, written in a cylindrical form:

$$
T=P \cdot R
$$

With $R$ the radius of the urethra. As the urethra does have a thick wall we calculated stress in units of force per unit surface by dividing Laplaces Law by the wall thickness $d$ (van Mastrigt et al. 1978):

$$
\sigma=\frac{T}{d}=\frac{P \cdot R}{d}
$$

With $\sigma$ stress in the urethral wall and $R$ the average of the inner and outer diameter of the urethra. The inner and outer diameter can be calculated from the injected volume $V_{i n j}$, the tissue-volume $V_{t}$ and the initial volume $V_{i}$. In the model urethras without a circular channel-profile $V_{i}=0$. With the inner and outer diameter after injection of the volume written in terms of injected, tissue and initial volumes Laplaces Law can be rewritten in terms of volumes, corrected for the state before injection of the volume:

$$
\sigma=\left(\frac{2 \cdot\left(V_{i n j}-\sqrt{V_{i}} \cdot \sqrt{V_{t}+V_{i}}+\sqrt{V_{i n j}+V_{i}} \cdot \sqrt{V_{i n j}+V_{t}+V_{i}}\right)}{V_{t}}\right) \cdot P
$$

The applied strain $\epsilon$ can be calculated from the change in average circumference of the cross-sections before and after injection. The average circumference is calculated from the averages of the inner and outer radius before $\left(R_{\text {avg }, 0}\right)$ and after $\left(R_{a v g}\right)$ injection and rewritten in terms of the above mentioned volumes:

$$
\epsilon=\frac{\ell-\ell_{0}}{\ell_{0}}=\frac{2 \pi R_{a v g}-2 \pi R_{a v g, 0}}{2 \pi R_{a v g, 0}}=\frac{\sqrt{V_{i}+V_{t}+V_{i n j}}+\sqrt{V_{i}+V_{i n j}}}{\sqrt{V_{i}+V_{t}}+\sqrt{V_{i}}}-1
$$


Using the rewritten Laplaces Law (Eqn. A-8) the coefficients $a_{n}(n=0,1,2)$ in Eqn. A-6 can be written in terms of stress. The time-constants $\tau_{1}$ and $\tau_{2}$ of the decay in stress can be calculated as the inverse of the coefficients $b_{m}(m=1,2)$. And the oscillation frequency $f$ is the coefficient $c_{2}$. 